\title{
Quasilinear stochastic elliptic equations with reflection: the existence of a density
}

\author{
SAMY TINDEL* \\ Département de Mathématiques, Institut Galilée, Université Paris 13, Avenue J.B. Clément, \\ 93430 Villetaneuse, France. E-mail: tindel@zeus.math.univ-paris13.fr
}

In this paper we prove the absolute continuity of the law of the solution to an elliptic stochastic partial differential equation with an additive white noise reflected at zero. The proof is based on Malliavin's calculus tools, and some methods of variational inequalities and ordinary partial differential equations driven by measure data.

Keywords: Malliavin's calculus; partial differential equations involving measure data; stochastic partial differential equations; variational inequalities

\section{Introduction}

In Nualart and Tindel (1995) we proved the existence and uniqueness of the solution to a quasilinear stochastic elliptic equation with reflection of the type

$$
-\Delta u(x)+f(u(x))=\dot{W}(x)+\eta, \quad x \in D,
$$

with Dirichlet-type boundary conditions, where $D$ is a bounded open domain of $\mathbb{R}^{k}$, $k=1,2,3, f$ is a continuous non-decreasing function and $\{\dot{W}(x) ; x \in D\}$ is a white noise on $D$. The solution is a pair $(u, \eta)$ where $u$ is a non-negative continuous random field on $D$ and $\eta$ is a random measure satisfying $\int_{D} u \mathrm{~d} \eta=0$. In this paper, we shall prove that the solution $u(x)$ has a density on $(0, \infty)$ for any $x \in D$, using the approximating sequence $u^{\epsilon}$ introduced by Nualart and Tindel (1995), and the classical tools of Malliavin's calculus.

Note that a similar problem has been treated by Lépingle et al. (1989) for the onedimensional case. As far as the existence and uniqueness are concerned, the parabolic case has also been studied by Nualart and Pardoux (1992) when the diffusion coefficient is constant, and by Donati-Martin and Pardoux (1993) in the case of a general diffusion coefficient. Donati-Martin and Pardoux (1995) showed a density result for the solution of the general parabolic problem with reflection. In this case, one of the main technical difficulties is due to the diffusion coefficient, which has to be removed in a certain way. However, the elliptic case is simpler, since we can only deal with an additive white noise (there is no existence and uniqueness result for a general diffusion coefficient), and we shall be able to prove the positivity of the norms of the derivatives $\left\|D u^{\epsilon}\right\|_{H}$ much more easily than was done by Donati-Martin and Pardoux (1995). 
This paper is organized as follows: Section 2 is dedicated to preliminary results on Malliavin's calculus, ordinary partial differential equations (PDEs) and stochastic partial differential equations (SPDEs) that we shall need later. Then, in Section 3, we shall prove the differentiability of the solution, and the existence of a density for the law of the solution on $(0, \infty)$.

\section{Preliminaries}

\subsection{General notation}

Let $D$ be an open bounded subset of $\mathbb{R}^{k}$, with $k \in\{1,2,3\}$. We shall consider a Gaussian family of random variables $\{W=W(B), B \in \mathscr{B}(D)\}$, defined in a complete probability space $(\Omega, \mathscr{F}, P)$ such that $\mathrm{E}(W(B))=0$ and

$$
\mathrm{E}(W(A) W(B))=|A \cap B|,
$$

where $|A \cap B|$ denotes the Lebesgue measure of the set $A \cap B$.

We shall call $H$ the space $L^{2}(D)$. The symbol $\Delta$ denotes the Laplace operator in $H$ and $\mathscr{C}_{k}^{\infty}(D)$ denotes the set of infinitely differentiable functions on $D$ with compact support included in $D$. We shall denote by $(\cdot, \cdot)$ the scalar product in $H$, and by $\|\cdot\|_{\infty}$ the supremum norm on $D$. We shall also denote by $\mathscr{C l}(D)$ the set of bounded measures on $D$, and by $W_{0}^{p, q}(D)$ the $(p, q)$ Sobolev space on $D$ with Dirichlet boundary conditions, i.e.,

$$
W_{0}^{p, q}(D)=\left\{f \in \mathscr{C}(\bar{D}) ; D^{i_{1} \ldots i_{m}} f \in L^{q}(D) \forall m \leqslant p, f_{\mid \partial D}=0\right\} .
$$

Let $G_{D}$ be the fundamental solution of the Poisson equation with Dirichlet boundary conditions. That is, for any $h \in H$,

$$
g(x)=\int_{D} G_{D}(x, y) h(y) \mathrm{d} y
$$

is the unique solution of

$$
\begin{aligned}
-\Delta g(x) & =h(x), \quad x \in D, \\
g_{\mid \partial D} & =0 .
\end{aligned}
$$

Let us recall that, by Donati-Martin (1992),

$$
\sup _{x \in D}\left\|G_{D}(x, .)\right\|_{H}=M \leqslant \infty .
$$

\subsection{Malliavin's calculus associated to the white noise in $D$}

With the notation given above, for any $h \in H$ we shall call $W(h)$ the Wiener integral on $h$ on $D$, i.e., 


$$
W(h)=\int_{D} h(x) W \mathrm{~d} x .
$$

Let us consider the space

$$
B=\left\{\omega \in \mathscr{C}(\bar{D}) ; \omega_{\mid \partial D}=0\right\}
$$

the function

$$
\begin{aligned}
i: H & \rightarrow B \\
h & \mapsto i(h)(x)=\int_{D} G_{D}(x, y) h(y) \mathrm{d} y
\end{aligned}
$$

and $u_{0}$ the process with paths in $B$ defined by

$$
u_{0}(x)=\int_{D} G_{D}(x, y) W \mathrm{~d} y=W\left(G_{D}(x, .)\right) .
$$

Then, if $\mu$ is the law of $u_{0}$, it has been shown by Donati-Martin (1992) that $(B, H, \mu)$ is an abstract Wiener space.

The space $\mathscr{S}$ of smooth functionals on $B$ will be the set of random variables of the form

$$
F=\ell\left(W\left(h_{1}\right), \ldots, W\left(h_{n}\right)\right),
$$

where $n \in \mathbb{N}, h_{i} \in H, \ell \in C_{b}^{\infty}\left(\mathbb{R}^{n}\right)$. For such a variable, we can define a derivative $D F$ as an $H$-valued random variable, by

$$
D_{z} F=\sum_{i=1}^{n} \frac{\partial l}{\partial x_{i}}\left(W\left(h_{1}\right), \ldots W\left(h_{n}\right)\right) h_{i}(z), \quad z \in D .
$$

For $p>1$, we shall denote by $\mathbb{D}^{1, p}$ the closure of $\mathscr{S}$ with respect to the seminorm

$$
\|F\|_{1, p}=\left\{\mathrm{E}\left[|F|^{p}\right]+\mathrm{E}\left[\|D F\|_{H}^{p}\right]\right\}^{1 / p} .
$$

We shall use the two following rules to get our density result (see Nualart (1995) for the demonstrations of this).

Theorem 2.1. Let $\left\{F_{n} ; n \geqslant 1\right\}$ be a family of elements of $\mathbb{D}^{1,2}$ converging to $F$ in $L^{p}(\Omega)$ for $p>1$. Suppose that $\left\{D F_{n} ; n \geqslant 1\right\}$ is a bounded family in $L^{p}(\Omega ; H)$. Then $F \in \mathbb{D}^{1, p}$, $F_{n} \in \mathbb{D}^{1, p}$ for every $n \geqslant 1$, and there exists a subsequence of $\left\{D F_{n} ; n \geqslant 1\right\}$ converging to $D F$ in the weak topology of $L^{p}(\Omega ; H)$.

Theorem 2.2. Let $F$ be a real random variable defined on $\Omega$. Suppose that $F \in \mathbb{D}^{1,2}$ and that there exists $A \in \mathscr{F}$ such that $\|D F\|_{H}>0$ a.s. on $A$. Then the measure $\left(\mathbf{1}_{A} P\right) F^{-1}$ is absolutely continuous with respect to the Lebesgue measure.

The following rule, due to (Sugita 1985), will ensure the differentiability of the solution to an elliptic SPDE. 
Theorem 2.3. Let $F \in L^{2}(\Omega)$. Then $F \in \mathbb{D}^{1,2}$ if and only if the two following conditions are verified.

(1) For all $h \in H$, there exists a version $\tilde{F}_{h}$ of $F$ such that, for all $\omega \in \Omega$, the mapping $t \mapsto \tilde{F}_{h}[\omega+t i(h)]$ is absolutely continuous.

(2) There exists $\eta \in L^{2}(\Omega ; H)$ such that, for all $h \in H$,

$$
P-\lim _{t \rightarrow 0} \frac{1}{t}\{F[\omega+t i(h)]-F(\omega)\}=(\eta(\omega), h) .
$$

\subsection{Ordinary elliptic partial differential equations}

We shall recall here some results given by Boccardo and Gallouët (1992). Consider the domain $D$ and the equation

$$
\begin{aligned}
-\Delta u(x)+g(x, u(x)) & =\lambda(x), \quad x \in D, \\
u_{\mid \partial D} & =0,
\end{aligned}
$$

where $g$ is a measureable function from $D \times \mathbb{R}$ to $\mathbb{R}$, and $\lambda \in \mathscr{L}(D)$. We say that $u$ is a weak solution to (2) if $u \in W_{0}^{1,1}(D), \Delta u \in L_{\mathrm{loc}}^{1}(D), g(u) \in L_{\mathrm{loc}}^{1}(D)$, and for any $\psi \in \mathscr{C}_{k}^{\infty}(D)$,

$$
-(u, \Delta \psi)+(g(u), \psi)=\int_{D} \psi(x) \lambda \mathrm{d} x,
$$

where $g(u)(x)$ stands for $g(x, u(x))$. Then the following result (which is a very particular case of those of Boccardo and Gallouët) holds.

Theorem 2.4. If $g: D \times \mathbb{R} \rightarrow \mathbb{R}$ is a measurable function such that

(1) $s \mapsto g(x, s)$ is continuous a.e. in $x \in D$,

(2) $g(x, s) s \geqslant 0$ for every $s \in \mathbb{R}$, a.e. in $x \in D$,

(3) $\sup \{|g(x, s)| ;|s| \leqslant t, x \in D\} \leqslant M(t)<\infty$ for every $t \in \mathbb{R}$ and

(4) there exist $b_{1}, b_{2}>0$ such that $|g(x, s)| \leqslant b_{1}+b_{2}|s|$ for any $s \in \mathbb{R}$, for almost any $x \in D$,

then there exists a solution $u$ to (2) for any $\lambda \in \mathscr{M l}(D)$.

Remark 1. In order to prove the existence part of the result, Boccardo and Gallouët use a sequence $\left\{\ell_{n} ; n \geqslant 1\right\}$ of functions of $W^{-1,2} \cap L^{1}(D)$ converging to $\lambda$ in the distribution sense. They call $u^{n}$ the solution of

$$
\begin{aligned}
-\Delta u^{n}(x)+g\left(u^{n}\right)(x) & =\ell_{n}(x), \quad x \in D, \\
u_{\mid \partial D} & =0 .
\end{aligned}
$$

Then they prove that 


$$
\begin{aligned}
\lim _{n \rightarrow \infty} u^{n}(x) & =u(x) \text { a.e. in } x \in D, \\
W_{0}^{1, q}-\lim _{n \rightarrow \infty} u^{n} & =u,
\end{aligned}
$$

for every $1 \leqslant q \leqslant k /(k-1)$, i.e., $q<\infty$ if $k=1, q<2$ if $k=2$ and $q<\frac{3}{2}$ if $k=3$.

Remark 2. Boccardo and Gallouët do not show the uniqueness of the solution to (2) in their general case. However, we shall apply the result of Theorem 2.4 when $g$ is an increasing function and, in this case, if $u$ and $v$ verify $u_{\mid \partial D}=v_{\mid \partial D}=0$ and

$$
\begin{aligned}
& -\Delta u(x)+g(u(x))=\lambda(x), \\
& -\Delta v(x)+g(v(x))=\lambda(x),
\end{aligned}
$$

then the difference $u-v$ verifies

$$
\begin{array}{r}
(-\Delta(u-v), u-v)=-(g(u)-g(v), u-v), \\
\|\nabla(u-v)\|_{H}^{2}=-(g(u)-g(v), u-v) .
\end{array}
$$

By positivity of $[g(u)-g(v)](u-v)$, the right-hand term of the above equality is negative, and thus $u=v$ a.e. on $D$, which proves the uniqueness of the solution.

\subsection{Elliptic stochastic partial differential equations}

Let $D$ be an open bounded subset of $\mathbb{R}^{k}$, with $k \in\{1,2,3\}$. We shall recall some results on a nonlinear stochastic elliptic equation with Dirichlet boundary condition of the type

$$
\begin{aligned}
-\Delta u(x)+f(u(x)) & =\dot{W}(x), \quad x \in D, \\
u_{\mid \partial D} & =0,
\end{aligned}
$$

where $\dot{W}(x)$ is the formal derivative of $W$ with respect to the Lebesgue measure, and $f: \mathbb{R} \rightarrow \mathbb{R}$ is a measurable function. We shall call $\mathrm{Eq}(f)$ such an equation, and assume from now the following hypothesis on $f$.

(H1) The function $f$ is a $\mathscr{C}^{1}$ function such that $f^{\prime}>0$.

The precise definition of the solution to (3) is given as follows.

Definition 2.1. A process $u$ is said to be a solution to (3) if

(1) $\{u(x), x \in \bar{D}\}$ is a continuous stochastic process on $\bar{D}$, with $u_{\mid \partial D}=0$ a.s. and

(2) for all $\phi \in \mathscr{C}_{k}^{\infty}(D)$ we have

$$
-(u, \Delta \phi)+(f(u), \phi)=\int_{D} \phi(x) W \mathrm{~d} x
$$


With the general notation introduced in Section 2.1, we can define the Gaussian random field

$$
u_{0}(x)=\int_{D} G_{D}(x, y) W \mathrm{~d} y .
$$

It has been shown by Buckdahn and Pardoux (1990) that $u_{0}$ has a version which is $\alpha$-Hölder continuous on $\bar{D}\left(\alpha<\frac{3}{8}\right.$ if $k=3, \alpha<1$ if $k=2$ and $\alpha=1$ if $\left.k=1\right)$. Moreover, $u_{0}$ satisfies the stochastic elliptic equation

$$
\begin{aligned}
-\Delta u_{0}(x) & =\dot{W}(x), \quad x \in D, \\
u_{0 \mid \partial D} & =0
\end{aligned}
$$

in the distribution sense. This means that, for every $\phi \in \mathscr{C}_{k}^{\infty}(D)$,

$$
-\left(u_{0}, \Delta \phi\right)=\int_{D} \phi(x) W \mathrm{~d} x \quad \text { a.s. }
$$

Then (3) is equivalent to its integral form

$$
u(x)+\int_{D} G_{D}(x, y) f(u(y)) \mathrm{d} y=\int_{D} G_{D}(x, y) W \mathrm{~d} y .
$$

In order to have a functional form of (4), we can introduce the map

$$
\begin{aligned}
T: B & \rightarrow B \\
\omega & \mapsto T(\omega)=\omega+i(f(\omega)) .
\end{aligned}
$$

Then (4) can be written

$$
T(u)=u_{0} .
$$

It has been shown by Donati-Martin (1992) that, if $f$ verifies (H1), then $T$ is bijective, which proves that (3) has a unique solution. Moreover, the transformation

$$
T(\omega)=\omega+i(f(\omega))
$$

verifies the hypothesis given in Theorem 6.2 of Kusuoka $(1982$, p. 583) in $(B, H, \mu)$. In particular, for every $x \in D$, the law of $u(x)$ has a density, and $f(\omega): B \rightarrow H$ is a $H-\mathscr{C}^{1}$ function. We also have the following differentiation rule.

Theorem 2.5. If $f$ verifies (H1), the solution $u$ to (3) is such that $u(x) \in \mathbb{D}^{1,2}$. The process $\left\{D_{z} u(x) ; z \in D\right\}$ verifies

$$
D_{z} u(x)+\int_{D} G_{D}(x, y) D_{z} u(y) f^{\prime}(u(y)) \mathrm{d} y=G_{D}(x, z) .
$$

Proof. We shall divide the proof into two steps. 
Step 1 Differentiability of $T^{-1}$. We proved in Tindel (1996) that $T^{-1}$ is a continuous function from $B$ to $B$. Let us prove now that $T$ is a differentiable function such that $L_{v}$, the differential of $T$ in $v$ is a non-degenerate linear function from $B$ to $B$, for any $v \in B$. Indeed, if $f$ verifies (H1), $T$ is a differentiable function of $v$ and for any $w \in B, L_{v} w$ is defined by

$$
L_{v} w(x)=w(x)+\int_{D} G_{D}(x, y) f^{\prime}(v(y)) w(y) \mathrm{d} y, \quad x \in D .
$$

Then it is easily seen that $L_{v}$ is non-degenerate; if $L_{v} w=0$, we have, in the non-integral form

$$
\begin{aligned}
-\Delta w+f^{\prime}(v(x)) w(x) & =0, \quad x \in D, \\
w_{\mid \partial D} & =0 .
\end{aligned}
$$

Since $f^{\prime}$ is continuous and positive, this equation verifies the hypothesis of Thereom 2.4 , and thus has a unique solution (cf. Remark 2 of that theorem), which is the null function. Moreover (Friedman 1975, p. 145), if $w$ is Hölder continuous, $L_{v}$ can be inverted as

$$
\left[L_{v}^{-1} w\right](x)=\tilde{\mathrm{E}}_{x}\left[\int_{0}^{\tau} w(\xi(t)) \exp \left(-\int_{0}^{t} f^{\prime}(v(\xi(s))) \mathrm{d} s\right) \mathrm{d} t\right],
$$

where $\xi$ is an $\mathbb{R}^{k}$-valued Brownian motion on a complete probability space $(\tilde{\Omega}, \tilde{\mathscr{F}}, \tilde{P}), \tau$ its exit time from $D$, and where $\tilde{\mathrm{E}}_{x}$ denotes the mathematical expectation with respect to $\tilde{P}$ with initial condition $x$ for $\xi$. In particular, if we set

$$
R=\sup _{x \in D} \tilde{\mathrm{E}}_{x}[\tau]
$$

we get $\left\|L_{v}^{-1}\right\| \leqslant R$ for every $v \in B$, by extension to a general continuous function $w$.

Step 2 Differentiability of $u(x)$. We shall apply Theorem 2.3 to $F(\omega)=u(x, \omega)$ for a fixed $x \in D$. From Step 1, we have almost surely

$$
\|u\|_{\infty}=\left\|T^{-1} u_{0}\right\|_{\infty} \leqslant R\left\|u_{0}\right\|_{\infty},
$$

which proves that $u(x) \in L^{2}(\Omega)$ for all $x \in D$, since $u_{0}$ is a Gaussian process. Let us denote by $G_{D} h$ the function

$$
G_{D} h(x)=\int_{D} G_{D}(x, y) h(y) \mathrm{d} y .
$$

With the same type of argument, for any $h \in H$,

$$
F[\omega+t i(h)]=\left\{T^{-1}\left[u_{0}(\omega)+t G_{D} h\right]\right\}(x)
$$

is an absolutely continuous function of $t$. We have also that, almost surely, $L_{u_{0}}$ is nondegenerate and $\left\|L_{u_{0}}^{-1}\right\| \leqslant R$. Thus, almost surely,

$$
\lim _{t \rightarrow 0} \frac{1}{t}\left\{F[\omega+t i(h)]-F(\omega)-L_{u_{0}}^{-1} G_{D} h\right\}=0 .
$$

Finally, by (1), 


$$
\left\|L_{u_{0}}^{-1} G_{D} h\right\|_{\infty} \leqslant\left\|L_{u_{0}}^{-1}\right\|\left\|G_{D} h\right\|_{\infty} \leqslant R M\|h\|_{H},
$$

which proves that, for a random variable $\eta \in L^{2}(\Omega ; H)$,

$$
L_{u_{0}}^{-1} G_{D} h=(\eta, h) .
$$

The last result that we shall need on elliptic SPDEs will be a comparison theorem given by Buckdahn and Pardoux (1990).

Theorem 2.6. Let $f, g: \mathbb{R} \rightarrow \mathbb{R}$ be locally bounded continuous and non-decreasing functions such that $f \leqslant g$. Let $u$ be the solution of $\mathrm{Eq}(f)$ and $v$ the solution of $\mathrm{Eq}(g)$. Then $u \geqslant v$ a.s.

\subsection{Elliptic stochastic partial differential equations with reflection}

Keeping the general notation of Section 2.1, let us consider the nonlinear stochastic elliptic equation with reflection and Dirichlet boundary condition of the type

$$
\begin{aligned}
-\Delta u(x)+f(u(x)) & =\dot{W}(x)+\eta, \quad x \in D, \\
u_{\mid \partial D} & =0,
\end{aligned}
$$

where the function $f$ is measurable. We shall call $\operatorname{Eq}_{r}(f)$ such an equation. A precise definition of the solution will be the following.

Definition 2.2. A pair $(u, \eta)$ is said to be a solution to (6) if

(1) $\{u(x), x \in \bar{D}\}$ is a non-negative continuous stochastic process on $\bar{D}$, with $u_{\mid \partial D}=0$ a.s.,

(2) $\eta \mathrm{d} x$ is a random measure on $D$ such that $\eta(K)<\infty$ for all compact subset $K \subset D$,

(3) for all $\phi \in \mathscr{C}_{k}^{\infty}(D)$ we have

$$
-(u, \Delta \phi)+(f(u), \phi)=\int_{D} \phi(x) W \mathrm{~d} x+\int_{D} \phi(x) \eta \mathrm{d} x
$$

and

(4) $\int_{D} u(x) \eta \mathrm{d} x=0$.

The main result of Nualart and Tindel (1995) is as follows.

Theorem 2.7. Assume that $f$ is locally bounded, continuous and non-decreasing. Then there exists a unique solution $(u, \eta)$ to $\operatorname{Eq}_{r}(f)$.

\section{Existence of a density}

We shall still suppose here that $f$ verifies (H1). In order to differentiate (in the sense of Malliavin's calculus) the approximating solution of $u^{\epsilon}$ of $u$, we have to get a smoother 
approximation than that constructed by Nualart and Tindel (1995). We shall use here the same method as Donati-Martin and Pardoux (1995), let $h$ be a $\mathscr{C}^{1}$ non-increasing function with bounded derivative such that

$$
\begin{gathered}
h(x)=0, \quad x \geqslant 0, \\
0<h(x)<x^{-}, \quad x<0,
\end{gathered}
$$

and set $f^{\epsilon}=f-h / \epsilon$ for every $0<\epsilon \leqslant 1$. We shall also call $u^{\epsilon}$ the unique solution to $\operatorname{Eq}\left(f^{\epsilon}\right)$.

Lemma 3.1. Let $u$ be the solution to $\mathrm{Eq}_{r}(f)$. Then almost surely, $u^{\epsilon}(x)$ increases as $\epsilon$ decreases to 0 for every $x \in D$, and $\lim _{\epsilon \rightarrow 0} u^{\epsilon}(x)=u(x)$. Moreover, for any $x \in D, p>1$, we have $L^{p}(\Omega)-\lim _{\epsilon \rightarrow 0} u^{\epsilon}(x)=u(x)$, and $\|u\|_{\infty} \in L^{p}(\Omega)$.

Proof. The sequence $u^{\epsilon}$ is increasing by the comparison with Theorem 2.6, and we can prove, as in Nualart and Tindel (1995), that $u^{\epsilon}$ converges to $u$ almost surely. To get the $L^{p}(\Omega)$ convergence, we only have to prove that $\left\|u^{\epsilon}\right\|_{\infty}$ is bounded in $L^{p}(\Omega)$, for any $p>1$ : set $z^{\epsilon}=u^{\epsilon}-u_{0}$, and $\hat{z}^{\epsilon}$ the solution to the deterministic equation

$$
\begin{aligned}
-\Delta \hat{z}^{\epsilon}(x)+f\left(\hat{z}^{\epsilon}(x)\right) & =\frac{1}{\epsilon} h\left(\hat{z}^{\epsilon}(x)\right), \quad x \in D, \\
\hat{z}_{\mid \partial D}^{\epsilon} & =0 .
\end{aligned}
$$

The process $z^{\epsilon}$ verifies

$$
\begin{aligned}
-\Delta z^{\epsilon}(x)+f\left(z^{\epsilon}(x)+u_{0}(x)\right) & =\frac{1}{\epsilon} h\left(z^{\epsilon}(x)\right), \quad x \in D, \\
z_{\mid \partial D}^{\epsilon} & =0 .
\end{aligned}
$$

Hence, as in Nualart and Tindel (1995), we have

$$
\left\|z^{\epsilon}-\hat{z}^{\epsilon}\right\|_{\infty} \leqslant\left\|u_{0}\right\|_{\infty}
$$

and thus

$$
\begin{aligned}
\left\|u^{\epsilon}\right\|_{\infty} & =\left\|u^{\epsilon}-u_{0}+u_{0}\right\|_{\infty} \\
& \leqslant\left\|z^{\epsilon}\right\|_{\infty}+\left\|u_{0}\right\|_{\infty} \\
& =\left\|z^{\epsilon}-\hat{z}^{\epsilon}+\hat{z}^{\epsilon}\right\|_{\infty}+\left\|u_{0}\right\|_{\infty} \\
& \leqslant\left\|\hat{z}^{\epsilon}\right\|_{\infty}+\left\|z^{\epsilon}-\hat{z}^{\epsilon}\right\|_{\infty}+\left\|u_{0}\right\|_{\infty} \\
& \leqslant\left\|\hat{z}^{\epsilon}\right\|_{\infty}+2\left\|u_{0}\right\|_{\infty},
\end{aligned}
$$

which implies that, for a constant $k_{p}>0$, 


$$
\begin{aligned}
\mathrm{E}\left[\left\|u^{\epsilon}\right\|_{\infty}^{p}\right] & \leqslant k_{p}\left(\mathrm{E}\left[\left\|\hat{z}^{\epsilon}\right\|_{\infty}^{p}\right]+\mathrm{E}\left[\left\|u_{0}\right\|_{\infty}^{p}\right]\right) \\
& =k_{p}\left(\left\|\hat{z}^{\epsilon}\right\|_{\infty}^{p}+\mathrm{E}\left[\left\|u_{0}\right\|_{\infty}^{p}\right]\right),
\end{aligned}
$$

since $\hat{z}^{\epsilon}$ is a deterministic function. Moreover, the sequence $\hat{z}^{\epsilon}$ is increasing towards the unique solution $\hat{z} \in \mathscr{C}(\bar{D})$ of the elliptic ordinary equation with reflection (Bensoussan and Lions 1978)

$$
\begin{aligned}
-\Delta \hat{z}(x)+f(\hat{z}(x)) & =\eta \mathrm{d} x, \quad x \in D, \\
\hat{z}_{\mid \partial D} & =0,
\end{aligned}
$$

which proves that, if $0<\epsilon \leqslant 1$, then $\left\|\hat{z}^{\epsilon}\right\|_{\infty} \leqslant\left(\left\|\hat{z}^{1}\right\|_{\infty} \vee\|\hat{z}\|_{\infty}\right)$, and that there exists a constant $K$ such that

$$
\sup _{0<\epsilon \leqslant 1}\left\|\hat{z}^{\epsilon}\right\|_{\infty} \leqslant K
$$

Finally, $(B, H, \mu)$ is an abstract Wiener space, and by Fernique's lemma (see, for example, Kuo (1976)), there exists a constant $\alpha>0$ such that

$$
\mathrm{E}\left[\exp \left(\alpha\left\|u_{0}\right\|_{\infty}^{2}\right)\right]<\infty
$$

In particular, $\left\|u_{0}\right\|_{\infty}$ has moments of any order, which ends the proof.

Using this approximating sequence, we shall be able to prove that $u(x)$ is an element of $\mathbb{D}^{1, \infty}=\bigcap_{p \leqslant 1} \mathbb{D}^{1, p}$.

Lemma 3.2. For every $x \in D, p>1, u(x) \in \mathbb{D}^{1, p}$, and there exists a subsequence of $D u^{\epsilon}(x)$ converging to $\mathrm{Du}(x)$ in $L^{p}(\Omega ; H)$.

Proof. For every $x \in D, p>1$, by Lemma 3.1, we know that

$$
L^{p}(\Omega ; H)-\lim _{\epsilon \rightarrow 0} u^{\epsilon}(x)=u(x)
$$

In order to apply the rule of Theorem 2.1, we have to verify that $u^{\epsilon}(x) \in \mathbb{D}^{1, p}$ and $\left\{D u^{\epsilon}(x) ; 0<\epsilon \leqslant 1\right\}$ is bounded in $L^{p}(\Omega ; H)$. Recall that, for any $0<\epsilon \leqslant 1, u^{\epsilon}(x) \in \mathbb{D}^{1,2}$ and that $D u^{\epsilon}(x)$ verifies (see (5)), for any $z \in D$,

$$
D_{z} u^{\epsilon}(x)+\int_{D} G_{D}(x, y)\left(f^{\prime}-\frac{h^{\prime}}{\epsilon}\right)\left[u^{\epsilon}(y)\right] D_{z} u^{\epsilon}(y) \mathrm{d} y=G_{D}(x, z)
$$

We have chosen $f$ and $h$ such that $f^{\prime} \geqslant 0, h^{\prime} \leqslant 0$. Hence $f^{\prime}-h^{\prime} / \epsilon \geqslant 0$ and is nondecreasing as $\epsilon \searrow 0$. Set

$$
D_{z} u^{\epsilon}(x)=Y_{z}^{\epsilon}(x), \quad\left(f^{\prime}-\frac{h^{\prime}}{\epsilon}\right)\left[u^{\epsilon}(x)\right]=\sigma_{\epsilon}(x) .
$$

Then (7) can be written 


$$
Y_{z}^{\epsilon}(x)+\int_{D} G_{D}(x, y) \sigma_{\epsilon}(y) Y_{z}^{\epsilon}(y) \mathrm{d} y=G_{D}(x, z),
$$

or, in its non-integral form,

$$
\begin{aligned}
-\Delta Y_{z}^{\epsilon}(x)+\sigma_{\epsilon}(x) Y_{z}^{\epsilon}(x) & =\delta_{z}(x), \quad x \in D, \\
Y_{z \mid \partial D}^{\epsilon} & =0,
\end{aligned}
$$

where $\delta_{z}$ denotes the Dirac measure in $z$. We shall prove that $0 \leqslant Y_{z}^{\epsilon}(x) \leqslant G_{D}(x, z)$ a.s.

Step $1 \quad Y_{z}^{\epsilon}(x) \geqslant 0$. Let $Y(x)$ be the solution of the ordinary elliptic PDE

$$
\begin{aligned}
-\Delta Y(x)+\sigma(x) Y(x) & =\delta_{z}(x), \quad x \in D, \\
Y_{\mid \partial D} & =0,
\end{aligned}
$$

with $z \in D, \sigma \in \mathscr{C}(\bar{D})$ (and thus bounded), $\sigma \geqslant 0$. Note that $Y_{z}^{\epsilon}(x)$ verifies these conditions with $\sigma(x)=\sigma_{\epsilon}(x)$. As in Theorem 2.4, Remark 1, take a sequence $\left\{f_{n} ; n \geqslant 1\right\}$ of Lipschitz functions converging to $\delta_{z}$ in the distribution sense. By positivity of the Dirac measure, we can also suppose that $f_{n} \geqslant 0$ for every $n \geqslant 1$. Let $Y^{n}$ be the solution of

$$
\begin{aligned}
-\Delta Y^{n}(x)+\sigma(x) Y^{n}(x) & =f_{n}(x), \quad z \in D \\
Y^{n}(x)_{\mid \partial D} & =0 .
\end{aligned}
$$

The function $g: D \times \mathbb{R} \rightarrow \mathbb{R}$ such that

$$
g(x, r)=\sigma(x) r
$$

verifies the conditions of Theorem 2.4, and thus $\lim _{n \rightarrow \infty} Y^{n}(x)=Y(x)$ for almost any $x \in D$, and

$$
W_{0}^{1, q}-\lim _{n \rightarrow \infty} Y^{n}=Y
$$

where $1 \leqslant q \leqslant k /(k-1)$. Moreover, as in the proof of Theorem 2.5, $Y^{n}$ has the representation

$$
Y^{n}(x)=\tilde{\mathrm{E}}_{x}\left[\int_{0}^{\tau} f_{n}(\xi(t)) \exp \left(-\int_{0}^{t} \sigma(\xi(s)) \mathrm{d} s\right) \mathrm{d} t\right],
$$

where $\xi$ is a $\mathbb{R}^{k}$-valued Brownian motion. From this expression, we get directly that $Y^{n}(x) \geqslant 0$, and by convergence of $Y^{n}(x)$, we obtain that $Y(x) \geqslant 0$ for almost every $x \in D$. In our random case, by continuity of $x \mapsto Y_{z}^{\epsilon}(x)$, we have that for a given $(z, \epsilon)$, almost surely, $Y_{z}^{\epsilon}(x) \geqslant 0$ for every $x \in D$.

Step $2 Y_{z}^{\epsilon}(x) \leqslant G_{D}(x, z)$. With the notation of Step 1, let $Y_{z}^{\epsilon, n}$ be the solution of

$$
\begin{aligned}
-\Delta Y_{z}^{\epsilon, n}(x)+\sigma_{\epsilon}(x) Y_{z}^{\epsilon, n}(x) & =f_{n}(x), \quad x \in D, \\
\left.Y_{z}^{\epsilon, n}\right|_{\delta D} & =0,
\end{aligned}
$$


and $Y_{z}^{0, n}$ the solution of

$$
\begin{aligned}
-\Delta Y_{z}^{0, n}(x) & =f_{n}(x), \quad x \in D, \\
\left.Y_{z}^{0, n}\right|_{\delta D} & =0 .
\end{aligned}
$$

With the representation (8), we get

$$
\begin{aligned}
& Y_{z}^{\epsilon, n}(x)=\tilde{\mathrm{E}}_{x}\left[\int_{0}^{\tau} f_{n}(\xi(t)) \exp \left(-\int_{0}^{t} \sigma_{\epsilon}(\xi(s)) \mathrm{d} s\right) \mathrm{d} t\right], \\
& Y_{z}^{0, n}(x)=\tilde{\mathrm{E}}_{x}\left(\int_{0}^{\tau} f_{n}(\xi(t)) \mathrm{d} t\right),
\end{aligned}
$$

and by positivity of $\sigma_{\epsilon}$, for every $0<\epsilon \leqslant 1, z, x \in D$, we have $Y_{z}^{\epsilon, n}(x) \leqslant Y_{z}^{0, n}(x)$. Moreover, for every $x \in D$,

$$
\begin{gathered}
\lim _{n \rightarrow \infty} Y_{z}^{\epsilon, n}(x)=Y_{z}^{\epsilon}(x), \\
\lim _{n \rightarrow \infty} Y_{z}^{0, n}(x)=G_{D}(x, z),
\end{gathered}
$$

which proves, by continuity of $Y_{z}^{\epsilon}(x)$, that a.s. $Y_{z}^{\epsilon}(x) \leqslant G_{D}(x, z)$ for every $x \in D$.

By Step 1 and Step 2, we get that almost surely, for any $\epsilon \in[0,1]$ of the form $\epsilon=1 / n$, with $n \geqslant 1$,

$$
\left\|D u^{\epsilon}(x)\right\|_{H} \leqslant\left\|G_{D}(x, .)\right\|_{H} .
$$

Hence, by inequality (1), almost surely,

$$
\sup _{0<\epsilon \leqslant 1} \sup _{x \in D}\left\|D u^{\epsilon}(x)\right\|_{H} \leqslant M
$$

and $D u^{\epsilon}(x) \in L^{\infty}(\Omega ; H)$, which proves that $u(x) \in \mathbb{D}^{1, \infty}$.

We can now prove the main result of this paper.

Theorem 3.1. Let $u$ be the solution of $\mathrm{Eq}_{r}(f)$, where $f$ verifies condition $(\mathrm{H} 1)$. Then for every $x \in D$, the restriction of the law of $u(x)$ to $(0, \infty)$ is absolutely continuous with respect to the Lebesgue measure.

Proof: By the rule of Theorem 2.2, we have to show that, if $x \in D, a>0$, and if we set $\Omega_{a}=\{\omega \in \Omega ; u(x, \omega) \geqslant 3 a\}$, then $\|D u(x)\|_{H}>0$ a.s. in $\Omega_{a}$. Let us call first $\Omega_{0}$ the set of convergence of $u^{\epsilon}$ towards $u$ in $D$, and set

$$
\Omega_{a, k}=\left\{\omega \in \Omega_{0} ; u(x, \omega) \geqslant 3 a, u^{\epsilon}(x, \omega) \geqslant 2 a, \forall \epsilon \leqslant \frac{1}{k}\right\} .
$$

We have $\Omega_{a}=\bigcup_{k \geqslant 1} \Omega_{a, k}$, and thus we only have to prove that $\|D u(x)\|_{H}>0$ almost surely on $\Omega_{a, k}$.

For $x \in D$, fix $\omega \in \Omega_{a, k}$. Then we have $u^{1 / k}(x) \geqslant 2 a$. Set then 


$$
\Omega_{a, k, j}=\left\{\omega \in \Omega_{a, k} ; u(y) \geqslant a, \forall y \text { such that }|y-x| \leqslant \frac{1}{j}\right\} .
$$

We have $\Omega_{a, k}=\bigcup_{j \geqslant 1} \Omega_{a, k, j}$, and we shall prove that $\|D u(x)\|_{H}>0$ almost surely on $\Omega_{a, k, j}$. For $\omega \in \Omega_{a, k, j}$, we call $B_{j}$ the ball of centre $x$ and radius $1 / j$. By the increasing property of $u^{\epsilon}$, for any $\epsilon<1 / k$, we shall also have $u^{\epsilon}(y) \geqslant a$, and hence $h^{\prime}\left(u^{\epsilon}(y)\right)=0$ for any $y \in B_{j}$.

Let us recall that $Y_{z}^{\epsilon}(y)=D_{z} u^{\epsilon}(y)$ is a positive process verifying the elliptic equation

$$
\begin{aligned}
-\Delta Y_{z}^{\epsilon}(y)+\left(f^{\prime}-\frac{h^{\prime}}{\epsilon}\right)\left[u^{\epsilon}(y)\right] Y_{z}^{\epsilon}(y) & =\delta_{z}(y), \quad x \in D, \\
\left.Y_{z}^{\epsilon}\right|_{\partial D} & =0 .
\end{aligned}
$$

For $\epsilon \leqslant 1 / k$, set

$$
v=\left.Y_{z}^{\epsilon}\right|_{B_{j}}, \quad \phi=\left.Y_{z}^{\epsilon}\right|_{\partial B_{j}} .
$$

Then $\phi \geqslant 0, \phi$ is continuous on $\partial B_{j}$ and $v$ verifies the equation

$$
\begin{aligned}
-\Delta v(y)+f^{\prime}\left(u^{\epsilon}(y)\right) v(y) & =\delta_{z}(y), \quad y \in B_{j}, \\
v(y) & =\phi(y), \quad y \in \partial B_{j} .
\end{aligned}
$$

As in Lemma 3.2, we shall approximate $\delta_{z}$ by a sequence of positive Lipschitz functions $f_{n}$, and $v$ by the function $v^{n}$ defined by

$$
\begin{aligned}
-\Delta v^{n}(y)+f^{\prime}\left(u^{\epsilon}(y)\right) v^{n}(y) & =f_{n}(y), \quad y \in B_{j}, \\
v^{n}(y) & =\phi(y), \quad y \in \partial B_{j} .
\end{aligned}
$$

Keeping the notation of Lemma 3.2, by the representation result given by Friedman (1975), we get that

$$
\begin{aligned}
v^{n}(y)=\tilde{\mathrm{E}}_{y}\left[\int_{0}^{\tau} f_{n}(\xi(t)) \exp \left(-\int_{0}^{t} f^{\prime}\left(u^{\epsilon}(\xi(s))\right) \mathrm{d} s\right) \mathrm{d} t\right] \\
+\tilde{\mathrm{E}}_{y}\left[\phi(\xi(\tau)) \exp \left(-\int_{0}^{\tau} f^{\prime}\left(u^{\epsilon}(\xi(s))\right) \mathrm{d} s\right)\right] .
\end{aligned}
$$

Furthermore, we have that

$$
u^{1}(x) \leqslant u^{\epsilon}(x) \leqslant u(x)
$$

and, by continuity of $f^{\prime}, u^{1}$ and $u$,

$$
\sup _{0<\epsilon \leqslant 1} \sup _{x \in D} f^{\prime}\left(u^{\epsilon}(x)\right)=L<\infty .
$$

Thus, since $\phi$ is a positive function, $v^{n} \geqslant \bar{v}^{n}$, where $\bar{v}^{n}$ is defined by

$$
\bar{v}^{n}(y)=\tilde{\mathrm{E}}_{y}\left(\int_{0}^{\tau} f_{n}(\xi(t)) \exp (-L t) \mathrm{d} t\right) .
$$


Passing to the limit in $n$, we get that $v \geqslant \bar{v}$, where $v$ is the solution to

$$
\begin{aligned}
-\Delta \bar{v}(y)+L \bar{v}(y) & =\delta_{z}(y), \quad y \in B_{j}, \\
\bar{v}(y) & =0, \quad y \in \partial B_{j},
\end{aligned}
$$

or in its integral form, for $y \in B_{j}$,

$$
\bar{v}(y)+L \int_{B_{j}} G_{B_{j}}(y, w) \bar{v}(w) \mathrm{d} w=G_{B_{j}}(y, z),
$$

where $G_{B_{j}}$ is the fundamental solution to the Poisson equation on $B_{j}$ with Dirichlet boundary conditions. In particular, for $y=x$,

$$
\bar{v}(x)=G_{B_{j}}(x, z)-L \int_{B_{j}} G_{B_{j}}(x, w) \bar{v}(w) \mathrm{d} w .
$$

Using (11), we can see that $0 \leqslant \bar{v}(y) \leqslant G_{B_{j}}(y, z)$ for any $y \in B_{j}$. Thus,

$$
\begin{aligned}
\bar{v}(x) & \geqslant G_{B_{j}}(x, z)-L \int_{B_{j}} G_{B_{j}}(x, w) G_{B_{j}}(w, z) \mathrm{d} w \\
& \geqslant G_{B_{j}}(x, z)-L M^{2},
\end{aligned}
$$

where $M$ is defined by (1). The function $G_{B_{j}}$ is infinite on the diagonal. Thus, for a deterministic neighbourhood $A$ of $x$ such that $A \subset B_{j}$ and $|A|>0$, we get $G_{B_{j}}(x, z)>2 L M$ for every $z \in A$. Hence, if $\omega \in \Omega_{a, k, j}$, for any $z \in A, 0<\epsilon \leqslant 1 / k$, we have $D_{z} u^{\epsilon}(x) \geqslant L M$.

Let us take now a subsequence of $D u^{\epsilon}(x)$, that we shall call again $D u^{\epsilon}(x)$, converging to $D u(x)$ in the weak topology of $L^{2}(\Omega ; H)$, and thus in the weak topology of $L^{2}\left(\Omega ; L^{2}(A)\right)$; for any $\varphi \in \mathscr{C}_{k}^{\infty}(A), X \in L^{2}(\Omega)$, we have

$$
\lim _{\epsilon \rightarrow 0} \mathrm{E}\left[X\left(D u^{\epsilon}(x), \varphi\right)\right]=\mathrm{E}[X(D u(x), \varphi)] .
$$

For any positive square integrable random variable $X$, and any $\varphi \in \mathscr{C}_{k}^{\infty}(A)$, we have that, almost surely on $\Omega_{a, k, j}$,

$$
X(\omega)\left(D u^{\epsilon}(x)(\omega), \varphi^{2}\right) \geqslant L M\|\varphi\|_{L^{2}(A)}^{2},
$$

for any $\epsilon \leqslant 1 / k$. Hence

$$
\mathrm{E}\left[1_{\Omega_{a, k, j}} X\left(D u(x), \varphi^{2}\right)\right] \geqslant \mathrm{E}\left[1_{\Omega_{a, k, j}} L M\|\varphi\|_{L^{2}(A)}^{2}\right],
$$

which means that, almost surely in $\Omega_{a, k, j}$, we have $D_{z} u(x)(\omega) \geqslant L M>0$ for almost any $z \in A$. Thus $\|D u(x)\|_{H}>0$ a.s. on $\Omega_{a, k, j}$.

\section{References}

Bensoussan, A. and Lions, J.L. (1978) Applications des Inéquations Variationnelles en Controle Stochastique. Paris: Dunod. 
Boccardo, L. and Gallouët, T. (1992) Nonlinear elliptic and parabolic equations involving measure data. J. Funct. Anal., 87, 149-169.

Buckdahn, R. and Pardoux, E. (1990) Monotonicity methods for white noise driven SPDEs. In M. Pinsky (ed.), Diffusion Processes and Related Problems in Analysis, Vol. I, pp. 219-233. Basel: Birkhäuser.

Donati-Martin, C. (1992) Quasi-linear elliptic stochastic partial differential equation. Markov property. Stochastics, 41, 219-240.

Donati-Martin, C. and Pardoux, E. (1993) White noise driven SPDEs with reflection. Probab. Theory Related Fields, 95, 1-24.

Donati-Martin, C. and Pardoux, E. (1995) EDPS réfléchies et calcul de Malliavin. To be published. Friedman, A. (1975) Stochastic Differential Equations and Applications New York: Academic Press. Kuo, H. (1976) Gaussian Measure on Banach Spaces. Berlin: Springer-Verlag.

Kusuoka, S. (1982) The non-linear transformation of Gaussian measure on Banach space and its absolute continuity. J. Fac. Sci. Univ. Tokyo, 29, 567-597.

Lépingle, D., Nualart, D. and Sanz, M. (1989) Dérivation stochastique des diffusions réfléchies. Ann. Inst. Henri Poincaré, 25, 285-305.

Nualart, D. (1995) The Malliavin Calculus and Related Topics. Berlin: Springer-Verlag.

Nualart, D. and Pardoux, E. (1993) White noise driven quasilinear SPDEs with reflection. Probab. Theory Related Fields, 93, 77-89.

Nualart, D. and Tindel, S. (1995) Quasilinear stochastic elliptic differential equations with reflection. Stochastic Processes Applications, 57, 73-82.

Sugita, H. (1985) On a characterization of the Sobolev spaces over an abstract Wiener space. J. Math. Tokyo Univ. 25-24, 717-725.

Tindel, S. (1996) Diffusion approximation for elliptic stochastic differential equations. In H. Korezlioglu, B. Oksendal and A.S. Üstünel (eds), Stochastic Analysis and Related Topics V: the Silivri Workshop, pp. 255-269. Basel: Birkhäuser.

Received March 1996 and revised March 1997 